\title{
Dispersive chiral approach to Meson-meson dynamics: Spectroscopy results for light scalars and precision studies
}

\author{
J. R. Peláez \\ Departamento de Física Teórica II, Universidad Complutense, 28040 Madrid, SPAIN
}

\begin{abstract}
Dispersive approaches provide model independent description of meson-meson scattering. We first review here the use of dispersion relations to obtain a model independent unitarization of Chiral Perturbation Theory amplitudes, that establish the existence of light scalar mesons and whose leading $1 / N_{c}$ behavior suggest they ahve a non $\bar{q} q$ dominant component. We also review the forward dispersion relation checks on conflicting experimental data and the resulting very precise $\pi \pi$ scattering scattering amplitudes.
\end{abstract}

Keywords: Chiral Perturbation Theory, Dispersive approach, Unitarization, ligt scalar mesons PACS: 11.30.Rd,11.55.Fv,12.39.Fe,13.75.Lb,14.40.-n

Over the last years there has been a renewed interest in meson-meson scattering. The reasons are that its low energy description is relevant for understanding the QCD vacuum, and that the description below $1.2 \mathrm{GeV}$ requires the existence of some poles in the amplitudes, related to light scalar mesons. Such resonances are the subject of a strong debate, first on their existence, that seems to be settling, but also on their nature.

Data on meson-meson scattering is obtained indirectly from other processes involving nucleons or the decays of other heavy mesons. Thus, very often the existing data on meson-meson amplitudes are extracted with strong model dependent assumptions (onepion exchange, absorption models, rescattering, pole extrapolations to define the initial meson-meson state, etc...), and are therefore plagued with systematic uncertainties much larger than statistical errors. There are also $K \rightarrow \pi \pi l v$ experiments, known as $K_{l 4}$ decays, where the pion phase shifts are extracted in a particular combination of isospin 0 and 1 , free of the previous systematic uncertainties and yield very precise and reliable results, but limited to invariant masses smaller than the kaon mass.

Concerning the theoretical description, we have two model independent approaches. On the one hand, we have Chiral Perturbation Theory (ChPT), which is the effective Lagrangian of QCD, written as an expansion in masses and derivatives of pions, kaons and etas, which are the Goldstone bosons of the spontaneous chiral symmetry breaking of QCD. The only caveat to this systematic expansion is that it can only be applied at low energies. On the other hand, it is possible to use the usual S-matrix constraints of causality, analyticity, unitarity, crossing, etc... to write dispersion relations for the different meson-meson channels. We review here how we have recently applied this approach to check the consistency of different data sets and to obtain a precise pion-pion scattering amplitudes in the whole energy range. Of course, both approaches can also be combined to obtain a model independent description of data that also incorporates the low energy chiral symmetry constraints (see. H. Leutwyler's talk on Roy equations

\section{CP892, Quark Confinement and the Hadron Spectrum VII} edited by J. E. F. T. Ribeiro

(C) 2007 American Institute of Physics 978-0-7354-0396-3/07/\$23.00 
in this conference). We review here how this has been done by means of the Inverse Amplitude Method (IAM), which has the advantage that only uses ChPT input in the dispersive integrals and therefore allows for a relation between the resulting fits and QCD. Since the IAM generates the light resonances that appear in scattering, it is then possible to study their nature in terms of QCD parameters, like the number of colors.

\section{THE INVERSE AMPLITUDE METHOD FROM DISPERSION THEORY}

We will review here how the one-channel Inverse Amplitude Method (IAM) $[1,2,3]$ for pion-pion scattering is obtained just by using ChPT up to a given order inside a dispersion relation. There are no further assumptions and therefore the approach is model independent and provides an elastic amplitude that satisfies unitarity and has the correct ChPT expansion up to that given order.

To fix ideas, let us consider the ChPT series for a pion-pion scattering partial wave amplitude of definite isospin $I$ and angular momentum $J$, namely, $t_{I J}=t_{I J}^{(2)}+t_{I J}^{(4)}+\ldots$ where $t_{2}=O\left(p^{2}\right), t_{4}=O\left(p^{4}\right)$ and $p$ stands for the pion mass or momentum. For the complete partial wave $t_{I J}(s)$, it is possible to write a dispersion relation

$$
t_{I J}(s)=C_{0}+C_{1} s+C_{2} s^{2}+\frac{s^{3}}{\pi} \int_{s_{t h}}^{\infty} \frac{\operatorname{Im} t_{I J}\left(s^{\prime}\right) d s^{\prime}}{s^{\prime 3}\left(s^{\prime}-s-i \varepsilon\right)}+L C\left(t_{I J}\right),
$$

that, for convenience, we have subtracted three times. Note we have explicitly written the integral over the right hand cut (or physical cut, extending from threshold, $s_{t h}$ to infinity) but we have abbreviated by $L C$ the equivalent expression for the left cut (from 0 to $-\infty$ ). We could do similarly with other cuts, if present, as in the $\pi K$ case.

We can also write dispersion relations for $t^{(2)}$ and $t^{(4)}$, but remembering that $t^{(2)}$ is a pure tree level amplitude and it does not have imaginary part nor cuts:

$$
t_{I J}^{(2)}=a_{0}+a_{1} s, \quad t_{I J}^{(4)}=b_{0}+b_{1} s+b_{2} s^{2}+\frac{s^{3}}{\pi} \int_{s_{t h}}^{\infty} \frac{\operatorname{Im} t_{I J}^{(4)}\left(s^{\prime}\right) d s^{\prime}}{s^{\prime 3}\left(s^{\prime}-s-i \varepsilon\right)}+L C\left(t_{I J}^{(4)}\right) .
$$

We now recall that unitarity, for physical values of $s$ in the elastic region implies:

$$
\operatorname{Im} t_{I J}=\sigma\left|t_{I J}\right|^{2} \Rightarrow \operatorname{Im} \frac{1}{t_{I J}}=-\sigma \Rightarrow t_{I J}=\frac{1}{\operatorname{Re} t_{I J}^{-1}-i \sigma},
$$

where $\sigma=2 p / \sqrt{s}$. Therefore, the imaginary part of the inverse amplitude is exactly known in the elastic regime. We can then write a dispersion relation like that in (1) but now for the auxiliary function $G=\left(t_{I J}^{(2)}\right)^{2} / t_{I J}$, i.e.,

$$
G(s)=G_{0}+G_{1} s+G_{2} s^{2}+\frac{s^{3}}{\pi} \int_{s_{t h}}^{\infty} \frac{\operatorname{Im} G\left(s^{\prime}\right) d s^{\prime}}{s^{\prime 3}\left(s^{\prime}-s-i \varepsilon\right)}+L C(G)+P C,
$$

where now $P C$ stands for possible pole contributions in $G$ coming from zeros in $t_{I J}$. It is now straightforward to expand the subtraction constants and use that $\operatorname{Im} t_{I J}^{(2)}=0$ 
and $\operatorname{Im} t_{I J}^{(4)}=\sigma\left|t_{I J}^{(2)}\right|^{2}$, so that $\operatorname{Im} G=-\operatorname{Im} t_{I J}^{(4)}$. In addition, up to the given order, $L C(G) \simeq-L C\left(t_{I J}^{(4)}\right)$, whereas $P C$ is of higher order and can be neglected. Thus

$$
\frac{t_{I J}^{(2) 2}}{t_{I J}} \simeq a_{0}+a_{1} s-b_{0}-b_{1} s-b_{2} s^{2}-\frac{s^{3}}{\pi} \int_{s_{t h}}^{\infty} \frac{\operatorname{Im} t_{I J}^{(4)}\left(s^{\prime}\right) d s^{\prime}}{s^{\prime 3}\left(s^{\prime}-s-i \varepsilon\right)}-L C\left(t_{I J}^{(4)}\right) \simeq t_{I J}^{(2)}-t_{I J}^{(4)} .
$$

We have thus arrived to the so-called Inverse Amplitude Method (IAM):

$$
t_{I J} \simeq t_{I J}^{(2) 2} /\left(t_{I J}^{(2)}-t_{I J}^{(4)}\right)
$$

that provides an elastic amplitude that satisfies unitarity and has the correct low energy expansion of ChPT up to the order we have used. It is straightforward to extend it to other elastic channels or to higher orders [3]. Note also that, by looking at (3), it seems that it can also be derived by replacing $\operatorname{Re} t_{I J}^{(-1)}$ by its $O\left(p^{4}\right)$ ChPT expansion. But, strictly speaking, (3) is only valid in the real axis, whereas our derivation allows us to consider the amplitude in the complex plane, and, in particular, look for poles of the associated resonances. Actually, already ten years ago [3], with the single channel IAM we were able to generate poles for the $\rho(770), K^{*}(892)$ and most interestingly, the controversial $\sigma$ (also called $f_{0}(600)$ ), without any model dependent assumptions.

Note that the above one-channel IAM derivation is model independent, and that contrary to a wide belief in the community contains a left cut and respects crossing symmetry up to, of course, the order in the ChPT expansion that has been used.

The confusion may come from the fact that the IAM has also been applied in a coupled channel formalism, for which there is still no dispersive derivation, and sometimes with furtehr approximations. Indeed one can arrive to (6) in a matrix form, ensuring coupled channel unitarity, just by expanding the real part of the inverse T matrix. For the coupled channel case different approximations to $R e T^{-1}$ have been used:

- The fully renormalized one-loop ChPT calculation of $R e T^{-1}$ provides the correct ChPT expansion in all channels, also with left cuts approximated to $O\left(p^{4}\right)[4,5]$. Indeed, using ChPT parameters consistent with previous determinations within standard ChPT, it was possible $[5,6]$ to describe below $1.2 \mathrm{GeV}$ all the scattering channels of two body states made of pions, kaons or etas. Simultaneously, this approach [6] generates poles associated to the $\rho(770), K^{*}(892)$ vector mesons, and the $f_{0}(980), a_{0}(980), \sigma$ and $\kappa$ (also called $K_{0}(800)$ ) scalar resonances.

- Originally [7], the coupled channel IAM was used neglecting the crossed loops and tadpoles. This approach is considerably simpler, and although it is true that the left cut is absent, its numerical influence was shown to be rather small, since the mesonmeson data are nicely described with very reasonable chiral parameters and generates all the poles enumerated above. Let us remark that this approximation keeps the s-channel loops but also the tree level up to $O\left(p^{4}\right)$, and that this tree level encodes the effect of heavier resonances, like the rho. Thus, contrary to some common belief, this approach still incorporates, for instance, the low energy effects of t-channel rho exchange.

- Finally, if one is interested in describing just the scalar meson-meson channels, it is possible to use just one cutoff (or even a dimensional regularization scale) that numerically mimics the combination of chiral parameters that appear in those scalar channels. This has become very popular, even beyond the meson-meson interaction realm, due to its great simplicity but remarkable success [8]. 


\section{NATURE OF LIGHT SCALARS FROM UNITARIZED CHPT}

One of the big advantages of the unitarization approaches described in the previous sections is that when they use the fully renormalized ChPT amplitudes, they therefore have the correct chiral and flavor symmetry structure, including both the spontaneous and explicit symmetry breaking. Furthermore we also have the correct dependence on QCD parameters like the number of colors, which is of particular interest, since there are sharp predictions on how the mass and width of $\bar{q} q$ resonances should behave in a large $N_{c}$ QCD expansion. In particular, $M \simeq O(1)$ whereas $\Gamma \simeq O\left(1 / N_{c}\right)$.

The $1 / N_{c}$ leading behavior of all ChPT parameters is known and model independent, so that they can be varied accordingly to study [9] the $N_{c}$ dependence of the amplitudes. In particular, we can study the $N_{c}$ behavior of all the poles generated in the IAM. It has been shown that both the mass and width of the vector mesons generated with the IAM follow remarkably well the expected $\bar{q} q$ behavior. However, light scalars do not follow a dominant $\bar{q} q N_{c}$ behavior [9], at least for $N_{c}$ not too far from real life, $N_{c}=3$. In figure 1 we illustrate these two different behaviors calculated at with the $O\left(p^{4}\right)$ IAM, for the $\rho K^{*}(892)$, and for the $\sigma$, and $\kappa$, whose poles can be obtained with the one-channel IAM, and therefore in a model independent way. Similar plots can be found for the other scalars in [9].

At this point it is worth noting that two-meson loop diagrams are subdominant at large $N_{c}$. Indeed, the above results imply that for vectors the meson loops play a small role in the cancellations that lead to poles in the amplitude, whereas for scalars such loop diagrams are very important at $N_{c}=3$. Since these diagrams become smaller and smaller one could wonder about the influence of higher order effects in scalars. Thus recently, we have performed [10] the full two-loop IAM analysis of the $\sigma$ channel confirming that, as it happened for the $O\left(p^{4}\right)$ amplitude, close to $N_{c}=3$ the sigma behaves rather differently than expected for a $\bar{q} q$ state, but that as the loop diagrams are suppressed, a subdominant $\bar{q} q$ behavior is recovered at larger $N_{c}$. Remarkably, this $\bar{q} q$ behavior arises slightly above $1 \mathrm{GeV}$, as it can be seen in Figure 1 (right plot). This seems to support the suggestion [11] that there is a non- $\bar{q} q$ scalar nonet below roughly $1 \mathrm{GeV}$, and another $\bar{q} q$ nonet above, but using a mode independent framework based on dispersive integrals, ChPT and the large $N_{c}$ QCD behavior.

\section{PRECISE AMPLITUDES FROM DISPERSION RELATIONS AND ROY EQUATIONS}

In the previous approach we used ChPT inside the integrals of partial wave dispersion relations. This has the advantage that we can control all the parameters of our input, and allow for their variation in order to understand the nature of poles or the dependence on certain QCD parameters. However ChPT does not necessarily give a good description of the integrands at high energies. Thus, even though we performed several subtractions to suppress the high energy regime we cannot claim to have very precise amplitudes, but a qualitative and most likely a semi-quantitative description.

There are however other approaches where one can use directly the data inside the integrals. Of course, now it is much harder, if possible at all, to interpret changes in 

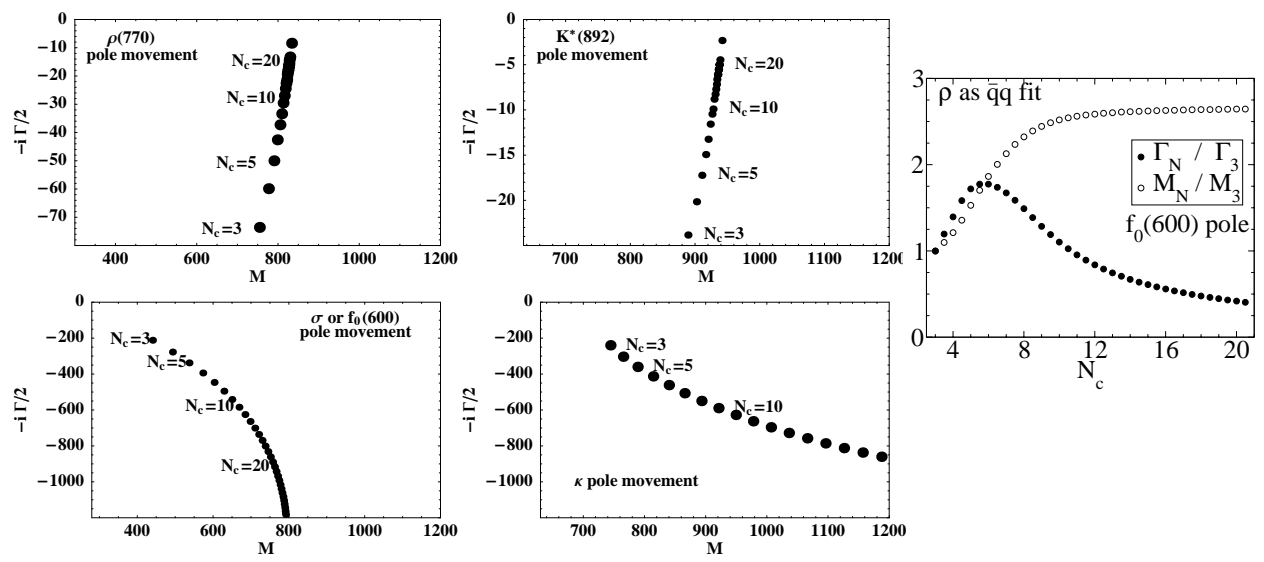

FIGURE 1. Leading $N_{c}$ behavior of resonance poles generated IAM. Left and center columns: with one-loop $O\left(p^{4}\right)$ ChPT Both the $\rho$ and $K^{*}$ poles in the imaginary plane behave as expected for $\bar{q} q$ states, namely $M \simeq O(1)$ and $\Gamma \simeq O\left(1 / N_{c}\right)$, respectively. In contrast, the light scalars $\sigma$ and $\kappa$ do not behave predominantly as $\bar{q} q$. Right: with two-loop $O\left(p^{6}\right)$ ChPT. We plot the mass $M$ and width $\Gamma$ evolution with $N_{c}$ calculated from the pole position, normalized to the $N_{c}=3$ case. Once more the dominant behavior is not that of a $\bar{q} q$ but a subdominant $\bar{q} q$ behavior emerges at larger $N_{c}$ around $1 \mathrm{GeV}$ in mass.

terms of parameters in terms of QCD, but we can get extremely precise results for the amplitude and other observables like scattering lengths, poles, etc... Recently, ChPT constraints and data have been included in single channel dispersion relations for $\pi \pi$ and $\pi K$ partial waves [12], confirming the existence of poles for $\sigma$ and $\kappa$ resonances, introducing some cutoffs on the dispersive integrals. For partial waves, the left cut is always a very delicate issue, because the large $t$ behavior is not well known. Two possible model independent approaches to this problem have been given in the literature: One is to rewrite the left cut integrals in terms of a coupled set of integral equations relating different partial waves, known as Roy equations for $\pi \pi$ scattering and RoySteininger equations for $\pi K$ scattering, which have been recently used to obtain precise determinations of the $\sigma[13]$ and $\kappa[14]$ poles, respectively.

Here I will comment on the other approach, namely, to use Forward Dispersion Relations (FDR), that is, to avoid using partial waves and use full amplitudes setting $t=0$. In a recent analysis we have used the following set of dispersion relations that form a complete isospin set: by choosing either $F=F_{00}$ or $F=F_{0+}$ in

$$
F(s)-F\left(4 M_{\pi}^{2}\right)=\frac{s\left(s-4 M_{\pi}^{2}\right)}{\pi} \text { P.P. } \int_{4 M_{\pi}^{2}}^{\infty} \mathrm{d} s^{\prime} \frac{\left(2 s^{\prime}-4 M_{\pi}^{2}\right) \operatorname{Im} F\left(s^{\prime}\right)}{s^{\prime}\left(s^{\prime}-s\right)\left(s^{\prime}-4 M_{\pi}^{2}\right)\left(s^{\prime}+s-4 M_{\pi}^{2}\right)} .
$$

we have two dispersion relations which are twice subtracted. Thus, the weight of the high energy part is quite suppressed, indeed as much as in Roy equations, but with the advantage of having always positive contributions to the integrand, a fact that makes them much more precise. In addition, by setting $s=2 M_{\pi}^{2}$, and $F=F_{00}$, we find two sum rules important to fix the Adler zeros. Finally, for the t-channel exchange of isospin 1, 



FIGURE 2. The data fits obtained in $[16,15]$ satisfy remarkably well the complete set of FDR and Roy Equations below $K K$ threshold and fairly well above. In this plots we compare the result of the direct parametrization (direct or "in" curves) versus the integral representation (dispersive or "out"). We emphasize these curves come from fits to data, without FDR or Roy equations as constraints, so that the agreement within uncertainties between continuous and dashed lines is even more remarkable.

which does not require subtractions, we use,

$$
F^{\left(I_{t}=1\right)}(s, 0)=\frac{2 s-4 M_{\pi}^{2}}{\pi} \text { P.P. } \int_{4 M_{\pi}^{2}}^{\infty} \mathrm{d} s^{\prime} \frac{\operatorname{Im} F^{\left(I_{t}=1\right)}\left(s^{\prime}, 0\right)}{\left(s^{\prime}-s\right)\left(s^{\prime}+s-4 M_{\pi}^{2}\right)} .
$$

At threshold this is known as the Olsson sum rule.

First of all, we have found [15] that only a few sets of data available in the literature, satisfy reasonably well the above forward dispersion relations within errors. Some of the most widely used experimental phase shift determinations indeed fail, on average for each FDR, by more than 2 standard standard deviations. The sets that satisfy FDR better are those closer to the fit using just the low energy data on $K_{l 4}$ decays commented in the introduction. All these sets have a marked "hunchback" in the 400 to $900 \mathrm{MeV}$ region that undoubtedly is caused by the $\sigma$ pole. We show in Figure 2 (upper row) the comparison between the FDR left hand sides ("direct" calculation) and FDR right hand sides ("dispersive" calculation). Note that the agreement below $K K$ threshold is astonishing given the tiny uncertainties and that only data has been fitted, not the FDR themselves. We have recently checked [18] that our direct fits to data also satisfy remarkably well Roy equations, as shown in Figure 2 (lower row).

In [15] we went further than this and improved the $\pi \pi$ amplitudes by constraining the fits to different partial waves to satisfy the FDR below $950 \mathrm{MeV}$. In this way we obtained an even more precise representation that lies not too far from the best fits to data only, precisely those showing the $\sigma$ "hunchback" in the S0 wave. 
Compared with the solution from Roy equations in [17], the phase shift solution that satisfy better the FDR representation in [15] agrees within errors in the low energy region for the $\mathrm{S}$ and $\mathrm{P}$ waves, but the $\mathrm{S} 0$ phase shifts above $400 \mathrm{MeV}$ lie about 2 standard deviations higher than in [17]. We also have a discrepancy of about two standard deviations in the D wave scattering lengths and the Regge residue of the $\rho$.

At present, and using our very recent improved fits above $K K$ threshold, we are working on an even more precise $\pi \pi$ amplitude by constraining our fits to satisfy simultaneously FDR and Roy equations, together with Froissart-Gribov and other sum rules. This parametrization will be used to obtain a precise determination of the $\sigma$ pole as well as of threshold parameters to determine the low energy ChPT constants.

In summary, I believe that, dispersive approaches combined with ChPT are the most powerful technique at our disposal to describe precisely and understand in terms of QCD the meson-meson interactions and light scalar spectroscopy.

\section{ACKNOWLEDGMENTS}

I thank the organization staff, and particularly E. Ribeiro, for creating such a nice working environment and offering me the chance to participate in this wonderful Conference.

\section{REFERENCES}

1. T. N. Truong, Phys. Rev. Lett. 61 (1988) 2526. A. Dobado, M. J. Herrero and T. N. Truong, Phys. Lett. B 235, 134 (1990).

2. A. Dobado and J. R. Pelaez, Phys. Rev. D 47, 4883 (1993)

3. A. Dobado and J. R. Pelaez, Phys. Rev. D 56, 3057 (1997)

4. F. Guerrero and J. A. Oller, Nucl. Phys. B 537, 459 (1999) [Erratum-ibid. B 602, 641 (2001)]

5. A. Gomez Nicola and J. R. Pelaez, Phys. Rev. D 65, 054009 (2002)

6. J. R. Pelaez, Mod. Phys. Lett. A 19, 2879 (2004)

7. J. A. Oller, E. Oset and J. R. Pelaez, Phys. Rev. Lett. 80, 3452 (1998). Phys. Rev. D 59, 074001 (1999) [Erratum-ibid. D 60, 099906 (1999)]

8. J. A. Oller and E. Oset, Nucl. Phys. A 620, 438 (1997) [Erratum-ibid. A 652, 407 (1999)]

9. J. R. Pelaez, Phys. Rev. Lett. 92, 102001 (2004)

10. J. R. Pelaez and G. Rios, arXiv:hep-ph/0610397. To appear in Phys. Rev. Lett.

11. E. Van Beveren, et al. Z. Phys. C 30, 615 (1986) and hep-ph/0606022. E. van Beveren and G. Rupp, Eur. Phys. J. C 22 (2001) 493, J. A. Oller and E. Oset, Phys. Rev. D 60 (1999) 074023. F. E. Close and N. A. Tornqvist, J. Phys. G 28, R249 (2002) (See. N.A. Tornqvist talk in this workshop)

12. Z. Y. Zhou and H. Q. Zheng, Nucl. Phys. A 775, 212 (2006). Z. Y. Zhou, et al.,JHEP 0502, $043(2005)$

13. I. Caprini, G. Colangelo and H. Leutwyler, Phys. Rev. Lett. 96, 132001 (2006) (See. H. Leutwyler's talk in this conference)

14. S. Descotes-Genon and B. Moussallam, arXiv:hep-ph/0607133.

15. J. R. Pelaez and F. J. Yndurain, Phys. Rev. D 71, 074016 (2005)

16. R. Kaminski, J. R. Pelaez and F. J. Yndurain, Phys. Rev. D 74, 014001 (2006) [Erratum-ibid. D 74, 079903 (2006)]

17. G. Colangelo, J. Gasser and H. Leutwyler, Nucl. Phys. B 603, 125 (2001)

18. R. Kaminski, J. R. Pelaez and F. J. Yndurain, in preparation. 
Copyright of AIP Conference Proceedings is the property of American Institute of Physics and its content may not be copied or emailed to multiple sites or posted to a listserv without the copyright holder's express written permission. However, users may print, download, or email articles for individual use. 